(Aus der Universitäts-Augenklinik Heidelberg [Direktor: Geh. Hofrat Professor Dr. Wagen $\operatorname{mann]}$.)

\title{
Weitere experimentelle Untersuchungen über die Quelle und den Verlauf der intraokularen Saftströmung.
}

\author{
2. Mitteilung. \\ Die Protoplasmastruktur der Ciliarepithelien als Kennzeichen ihrer \\ physiologischen Funktion. \\ Von \\ Professor Dr. Erich Seidel, \\ Oberarzt der Klinik.
}

Mit 2 Textabbildungen und 9 Abbildungen auf Tafel III.

Die häufig schon aufgeworfene Frage, ob der Vorgang bei der physiologischen Kammerwasserabsonderung eine physikalische Filtration bzw. Transsudation oder eine vitale Sekretion darstellt, wurde von Leber mit Bestimmtheit dahin beantwortet, daß vom chemischen Standpunkt aus entscheidende Gründe dagegen sprechen, im Vorgang der physiologischen Kammerwasserabsonderung eine Sekretion zu erblicken, da die Augenflüssigkeit sowohl bezüglich ihrex chemischen Zusammensetzung, sowie ihrer physikalischen Eigenschaften (osmotischer Druck) die Annahme einer Beteiligung besonderer vitaler Zellkräfte bei ihrer Absonderung nicht erfordert.

Die von verschiedenen Seiten gegen diese physikalische Auffassung der Kammerwasserbildung zugunsten einer vitalen Sekretionstheorie geäußerten Bedenken, die sich auf den angeblich gegenüber dem Blutserum erhöhten osmotischen Druck und den etwas höheren. Kochsalzgehalt des Kammerwassers stützten ${ }^{1}$ ), haben sich nicht als stichhaltig

1) Vgl. dazu die Ausführungen v. d. Hoeves (v. Graefes Archiv 82, 58. 1912), der auf Grund der bisher vorliegenden sowie seiner eigenen Versuchsergebnisse zu dem Schluß kommt: „Bis jetzt bringen also die physikaliseh chemischen Untersuchungen keinen Beweis gegen $L \in b$ ers Theorie, da $B$ Augenflüssigkeit ein Transsudat ist. " - Weis \& (Zeitschr. f. Augenheilk. 25, 4) wies auf die Mogliohkeit hin, den etwas erhöhten Kochsalzgehalt des Humor aqueus $(0,7-0,8 \%)$ gegenüber dem Blutserum $(0,6 \%)$ durch Diffusion aus der stärker kochsalzhaltigen Tränenflüssigkeit $(1,3 \%)$ durch die Hornhaut hindurch in der Vorderkammer zu erklären. Da aber das Kammerwasser infolge seines minimalen Eiweißgehaltes gegenüber dem Blutserum hypotonisch sein würde (bei gleichem Salzgehalt), so kann der erhöhte Kochsalzgehalt auch ohne weiteres selkundär, physikalisch durch Diffusion aus den Blutgefäßen (der Iris) ins Kammerwasser gelangt sein, wofür die Tatsache spricht, daß wir einen ähnlich gegenüber dem Blutserum erhöhten Salzgehalt im Liquor cerebrospinalis finden. 
erwiesen und auch die auf den ersten Blick mit der Annahme einer physikalisehen Transsudation des Kammerwassers schwer zu vereinbarende Tatsache des fast völligen Zurückgehaltenwerdens gewisser ins Blut gebrachter krystalloider Stoffe (Fluorescein) durch das intram okulare Sekretionsorgan vermag uns die moderne Kolloidchemie durch physiko-chemische Gesetze zu erklären ${ }^{1}$ ).

Obgleich man nun aus der Tatsache, daß ein Ábsonderungsprodukt blutfremde chemische Bestandteile enthält oder dem Blut nicht zukommende physikalische Eigenschaften aufweist, mit Sicherheit auf die Mitwirkung spezifischer vitaler, d.h. physiko-chemisch uns zur Zeit noch nicht erklärbarer Zellkräfte beim Absonderungsvorgang schließen kann, so ist anderseits aus dem Fehlen derartiger neuer, d. h. rom Blutserum abweichender Eigenschaften im Absonderungsprodukt eine aktive Mitbeteiligung lebender Zellen beim Absonderungsvorgang nicht auszuschließen, da ein durch physikalische Kräfte erreich barex „Arbeitseffekt" nicht notwe ndigerweise auch hierdurch zustande gekommen sein braucht, sondern ebensogut durch vitale oder auch durch das Zusammenwixken vitaler und physikalischer Kräfte erzielt worden sein kann. Die Frage nach der Beteiligung vitaler Zellkräfte beim Absonderungsvorgang des Kammerwassers kann somit nicht aus der chemischen Analyse des Selkretionsprodultes allein beantwortet werden.

Da wir nun wissen, daß flüssigkeitsausscheidende Zellen charakteristische morphologische Struktureigentuimlichkeiten ihres Protoplasmas zeigen, aus denen auf physiologische Zellvorgänge geschlossen werden kann, so mußten geeignete anatomische Untersuchungen imstande sein,

I) Leber hat schon vor Jahren bezüglich dieser Frage auf die Eigensohatt von Kohlenpulver hingewiesen, Farbstoffe (und auch andere Stoffe) aus ihren Lösungen auszuziehen und festzuhalten durch sog. Adsorption. Inzwischen hat die Kolloidchemie die große Bedeutung dieser Erscheinung der Adsorption oder Oberflächenwirkung gerade bei den Enaulsionskolloiden (Blutplasma) nit ihrer gewaltigen Oberflächenentwicklung immer mehr erkant und uns weiter gelehrt, daß gerade Proteine zu solchen relativ festen, aber doch leicht, "reversiblen ${ }^{6 *}$ Anlagerungen mit Salzen neigen (vgl. Wo. Ostwald, Grundriß der Kolloidchemie, desgl. Oppen b eimer, Biochemie). Daß eine ,oberfiächliche ${ }^{6}$ Bindung awischen Fluorescein und Eiweil tatsächlich eintritt, hat Friedmann experimentell bexiesen (Sitzungsbericht der Phys. Ges. Berlin 1909 [Med. Klin. 1909, Nr. 34]), wodurch das fast vollige Zurückgehalterwerden intravenôs injizierter Fuoresceinlösung im Ehrlichschen Experiment physikalisch erklärt sein würde.

Dex Ubertritt des Farbstoffes in das Nierensekret weist ant dis vitale Fuhigkeit der sezernierenden Nierontellen hin, die Bindung zu losen, was ja durohaus mit der Funiktion der Niere als asmo-regulatorisches Gieichgewichtorgan in Finklang steht. Auf den fundamentalen Untersohied zwischen Miere als Exkretionsorgan und gewissen Drüsen, die Sekretionsorgane darstellen vind die den Farbstoff nioht oder nur in mi nimalen Mengen (Speicheldrüse, Tränendrise, Plexus ohorioideus, Corpus ciliare) in ihr Absonderungsprodulat itbertreten lassen, babe ich frither sohon hingewiesen. 
die physiologische Frage nach der Mitbeteiligung vitaler Zellkräfte beim Absonderungsvorgang des physiologischen Kammerwassers zu entscheiden.

Der Nachweis vom Vorhandensein derartiger durch ihre Protoplasmastruktur auf physiologische sekretorische Funktion hindeutender Zellen im Auge mußte zugleich von neuem die umstrittene Hauptfrage der Ernährungsphysiologie des Auges beantworten, ob überhaupt das Kammerwasser im Auge eine Erneuerung infolge Zuflusses und Abflusses unterliegt, und die Lokalisation dieser Zellen im Auge konnte weiter die bekannte Streitfrage entscheiden, ob der Ciliarkörper oder die Irisvorderfläche als physiologisches Quellgebiet des Kammerwassers zu betrachten ist.

Verschiedene von mir bereits gemachte experimentelle Beobachtungen wiesen deutlich auf die aktive Mitbeteiligung der Ciliarepithelien beim Absonderungsvorgang des Kammerwassers hin, so daß in diesen Zellen eine charakteristische, auf Sekretion hindeutende Protoplasmastruktur in erster Linie zu erwarten war.

Bekanntlich ist schon wiederholt die Ansicht vertreten worden, daß das Epithel des Ciliarkörpers gleichsam ein sezernierendes Drüsenepithel darstelle und eine wesentliche Rolle bei der Kammerwasserabsonderung spiele (M éry, Boucheron, Treacher Collins, Nicati, Sattler), ohne daB jedoch für diese Behauptung nach Leber (Zirkulations- und Ernährungsverhältnisse des Auges, 1903, S. 251f.) Beweise erbracht worden wären.

Da aber doch Eiweiß nur in Spuren $(1 / 40 \%)$ ins physiologische Kammerwasser übertritt, so sind alle diejenigen, die eine Beteiligung des Ciliarepithels bei der Kammerwasserabsonderung leugnen, zu der m. E. unwahrscheinlichen Annahme gezwungen, daß das physiologische Produkt der Ciliarkörpercapillaren sich chemisch fundamental von dem der übrigen Körpercapillaren unterscheidet, das bekanntlich etwa 3\% Eiweiß (Lymphe) enthält, so daß sich (bei anatomischer Gleichheit) eine physiologische Sonderstellung der intraokularen Capillaren gegenüber den übrigen Körpercapillaren hieraus ergeben würde.

Da ich feststellen konnte, daß nach dauernder operativer Herabsetzung des intraokularen Drucks durch Elliotsche Trepanation bei Katzen das zunächst stark eiweißhaltige Kammerwasser nach etwa 10-14 Tagen wieder einen in physiologischen Grenzen liegenden Eiweißgehalt aufwies, trotz abnorm tiefen Augendrucks (Schiötz: $\frac{5,5}{15}$ $=5 \mathrm{~mm} \mathrm{Hg}$ ), so kann der intraokulare Gegendruck nicht zur Erkiärung des gegenüber der Körperlymphe so erheblich verminderten Gehaltes an Eiweiß des Humor aqueus herangezogen werden, ganz abgesehen, daß das Sekretionsprodukt der Plexus chorioidei des Gehims, die ent- 
wicklungsgeschichtlich den Ciliarfortsätzen gleichstehen und die physiologischerweise gegenüber einem viel geringeren Drucke arbeiten, als er im Auge herrscht, fast dieselbe chemische Zusammensetzung aufweist, wie der physiologische Humor aqueus.

Da ich nun weiter an vorher völlig intaktem Auge (Katze, Kaninchen), an mikroskopischen Schnitten vorher mit Osmium- und Chromsäure behandelter Präparate eiweißhaltige Lymphe im Innern der Ciliarfortsätze außerhalb der Blutcapillaren gefunden habe (vgl. Abb. I auf Tafel III) ${ }^{1}$ ), so muß die Frage, ob die Capillarendothelien oder die Ciliarepithelien den Eiweißübertritt ins Kammerwasser verhindem, dahin beantwortet werden, daß den Ciliarepithelien diese Fähigkeit zukommt, so daß auch diese Beobachtung wieder auf die aktive Beteili. gung des Ciliarepithels beim Absonderungsvorgang des Kammerwassers hinweist, wie das in ähnlicher Weise die von mir mit vitalen Farbstoffen erhaltenen miskroskopischen Versuchsergebnisse taten, worüber ich mich ja kürzlich erst eingehend. geäußert habe.

Nun ist der Epithelüberzug des Corpus ciliare schon wiederholt auf morphologische (bei einer sekretorischen Funktion zu erwartenden) Struktureigentümlichkeiten untersucht worden, und Henderson und Lane Cla y po ${ }^{2}$ ) kommen auf Grund ausgedehnter, mit sehr geeigneten histologischen Methoden vorgenommenen Untersuchungen (1907) zu dem Ergebnis, das Bestehen solcher Einrichtungen im Ciliarepithel glatt zu verneinen. Auf diese negativen Befunde stützt sich nun WeiB (1910 und 1911 $)^{9}$ ), indem er in ihnen einen Beweis gegen die Richtiglkeit von Lebers Auffassung über die sekretorische Funktion des Ciliarkörpers erblickt, ohne jedoch die bereits kurz vorher (1908 und 1910) bekanntgegebenen Befunde von $\mathrm{Mawa} \mathrm{s}^{\frac{4}{4}}$ zu erwähnen, der zu positiven Resultaten - Granulierung der Zellen - gelangte.

1) Abb. I auf Tafel III stellt zwei nebeneinander liegende Ciliarfortsätze eines völlig intakten Auges (Katze, Meth. So hridde) dar, von dem der rechte von einer homogenen (durch Osmium) leicht angedunkelten homogenen Masse offenbar eiweißhaltiger Lymphe angefibllt ist, die in dem linken micht festzustellen war (C. Zeiß, Objektiv A, Ocular 3). Es scheint demnach ein funktioneller physiologischer Unterschied zwischen den einzelnen Ciliarfortsätzen za bestehen, ähnlioh wie ein soloher zwischen den einzelnen Tubuli contorti bei vitaler Färbung der Niere hervortritt, woraus man auf ein "schichtweises" Arbeiten dieser Gebilde geschlossen hat.

2) Henderson und Lane Claypon, Ophth. Hosp. Rep. 1908, Study of the oillary Epithelium after pucture of the anterior Chamber.

3) Wei B, Der intraoculare Fussigkeitswechsel. Referat gehalten aut d. Tag. d. Vers. Deutscher Aaturf. u. Arzte za Königsberg 1. Pr. 1910; vgl Erweiterte Wiedergabo dieses Referates. Zeitsohr. f. Augenheill. 25, 4. 1911.

4) Mawas, Sur la structure de la rétine ciliaire. Compt. rend. de l'Acad. des Sc. 1908. Etude cytologique et physiologique sur la rétine ciliaire des Mammipères. Arch. d'Anat. micr. 12. 1910. 
Allerdings konnte Magitot ${ }^{1}$ ) die Beweiskraft der Befunde von Mawas für eine sekretorische Funktion der Ciliarepithelien nicht bestätigen, da er, ohne das Vorhandensein mitochondraler Protoplasmabildungen zu leugnen, dennoch das Ciliarepithel als aus völlig homogenen Zellen bestehend abbildet ${ }^{2}$ ) und ausdrücklich betont, keinerlei für eine sekretorische Funktion sprechenden Gebilde, wozu er in erster Linie Sekretvakuolen rechnet, mikroskopisch gefunden zu haben, so daß er geradezu das Ciliarepithel als ein ruhendes, inaktives Epithel dem aktiven, sekretorische Zelltätigkeit zeigenden Epithel des Aderhautgeflechts des Gehirns gegenüberstellt.

Bevor ich über die von mir erhobenen histologischen. Befunde berichte, sei zunächst kurz daran erinnert, daß für alle mit sekretorischen Eigenschaften begabten Zellen ganz bestimmte morphologische Struktureigentümlichkeiten geradezu charakteristisch sind, die in der Hauptsache darin bestehen, daß in ihrem Protoplasma mit bestimmten spezifischen histologischen Methoden zahlreiche, meist zu Fäden oder Ketten angeordnete feine Körner nachweisbar sind, die etwa parallel der Längsrichtung der Zelle verlaufen und die man als Fadenkörner oder Mitochondrien bezeichnet ${ }^{3}$ ).

Diese körnigen Gebilde im Zellprotoplasma werden von manchen Forschern (Renaut) auch Eklektosomen genannt, in dem Sinne, daB man ihnen die Fähigkeit elektiver Aufnahme, Fixierung und Konzentration von Nährsubstanzen, Giften und Arzneimitteln und die Ausarbeitung der Sekrete zuschreibt.

W. „Die neue Mitochondrienforschung tritt mit überwiegender Mehrheit für die Beteiligung der Mitochondrien am Sekretionsgeschäft ein. Stäbchenförmige Chondriokonten bilden das Sekret in Granula aus, die isoliert und ausgestoßen werden. Die Mitochondrien wären demnach

1) Magitot, L'humeur aqueuse et son Origine. Ann. d'Oculist. 1917. Magi * tot vermißte in den Ciliarepithelien das Vorkommen von Vakuolen. Solche Sekretvakuolen sind aber in hinlänglich dünnen Schnitten $(3 \mu)$, wie die folgenden Bilder zeigen werden, reichlich vorhanden und sind außerdem kein unbedingtes Erfordernis für eine sekretorische Zellfunktion, da man doch granuläre Formen der Sekretion kennt. (Über die Bedeutung der Vakuolen vgl. P. Ernst in Krehl-Marschands Handb. d. allgem. Pathologie III. I. S. 274, 362, 370).

2) 1. c. Tafel IV, Abb. 1.

3) Diese Granula (Mitochondrien) kommen in allen Körperzellen mit a k tiver Tätigkeit vor und stellen Stoffe dar, die für die Funktion der betreffenden Zelle gebrancht werden. Diese Zellfunktion besteht beider quergestreiften Muskelfaser in Kontraktion, in der Drüsenzelle in Sekretion. Es ist daher nicht angängig, aus dem Vorhandensein von Mitochondrien in Muskelzellen die funktionelle Bedeutung dieser Gebilde in Epithelzellen zu bezweifeln, wie das Magitot tut. (Vgl. dariber Duesberg, Plastosomen, "Apparato reticolare interno" und Chromidialapparat. Ergebnisse d. Anatomie u. Entwicklungsgeschichte Bd. XX. II. S. 812.)

v. Graefes Arehiv für Ophthalmologie. Bd. 102 . 
vegetative Organe, deren Hauptaufgabe in der Speicherung und Umbildung des aus dem Blute geschöpften Materials besteht. Je nach dem Sekretionszustand trifft man Körner (Mitochondrien), Fäden (Chondriomiten) oder Stäbchen (Chondriokonten), die aber alle in denselben Formenkreis zusammengehören. Sie werden zu Sekret aufgebraucht; je mehr Sekret ausgebildet wird, desto weniger Mitochondrien findet man. Die Fäden verarbeiten die Stoffe zu Granula" "1) (d. h. zu Sekrettropfen).

Diese Gebilde sind in einigen Drüsen, z. B. in der Niere, in den Zellen der Tubuli contorti als Heidenhainsche Stäbchen, sowie in den Zellen der Ausführungsgänge der Speicheldrüsen als Pflügersche Stäbchen schon lange bekannt, da sie hier mit den gewöhnlichen histoIogischen Methoden und besonders in der Niere mit allen bekannten vitalen Farbstoffen sehr leicht sichtbar zu machen sind. Da in den meisten anderen Drüsenzellen die ijblichen histologischen Methoden zu ihrer Darstellung nicht genügen, und sie sich auch hier gegen die meisten vitalen Farbstoffe vollkommen refraktär verhalten ${ }^{2}$ ), so wurde ihre Verbreitung und allgemeine biologische Bedeutung erst viel später erkannt, nachdem zunächst Altmann und darauf Benda duroh bestimmte Fixierungsverfahren und komplizierte Färbemethoden den Weg gezeigt hatten, sie überall, wo vorhanden, zur Anschaung zu bringen.

Da nun diese Mitochondrien, um die Worte Duesbergs ${ }^{3}$ ), eines weiteren hervorragenden Mitochondrienforschers, zu gebrauchen, im erwachsenen. Gewebe ohne jeden Zweifel und in größerer Menge nur in solchen Zellen vorkommen, welche durch ,,beträchtliche Aktivität im Nahrungsaustausch, dessen Sitz sie sind, bestimmt mit den Drüsenzellen verglichen werden können.", und alle Autoren über die Tatsacho völlig einig sind, daß stets mit einer Vermehrung der Sekretköme" eine beträchtliche Verringerung der Zahl der Mitochondrien eintritt, was sich nur aus einer Umbildung dieser Mitochondrien in Sekretkörner deuten läßt, so geht die allgemeine Auffassung dahin, daß da, wo ein ausgesprochener Mitochondralapparat in Epithelzellen vorhandeu ist, auch eine produktive Zelltätigkeit, eine Sekretion stattfinden muß, und daß umgekehrt das Fehlen eines Chondrioms, wie man die Gesamtheit der Mitochondrien in einer Zelle bezeichnet, eine sekretorische Funktion ausschließen läßt.

1) P. Ennst, Lebensersoheinungen als Maßstab für die Protoplasmastruktur. Verhandl. des naturw.-mediz. Vereins, Heidelberg, Bd. 13, Heft 1. 1914. Vgl. weiter P. Ernst, ,Pathologie der Zelle“. Krehl-Marchand, Handb. d. allgem. Pathologie Bd. III I; besonders Kapitel III (Übersicht über die neuen Theorien d. Protoplasmastruktur, S. 49). Kapitel XI. (Normale und pathologische Sekretionsvorgänge $S, 246$ ).

2) Duesberg, 1. c. S. 607,611 . u. 790 .

s) Duesberg, 1. c. S. 788 . 
Ich habe bei Katzen und albinotischen Kaninchen und bei der Gans das Ciliarepithel, die Irisvorderfläche, das Epithel der Hornhaut sowie den Plexus chorioideus im frischen Zustand sowie nach Behandlung nach den versehiedenen mitochondralen Methoden nach $\operatorname{Regaud}^{\mathbf{1}}$, Schridde ${ }^{2}$ ) und 0. Schulze $^{3}$ ) untersucht, wobei ich so verfuhr, da $B$ ich Tris und Ciliarkörper im Zusammenhang ließ, so daß man auf demselben Schnitte stets beide Gebilde beurteilen konnte.

Ich fand, daB sowohl im frischen Präparat, als auch an den nach den verschiedenen Methoden behandelten Schnitten die Epithelzellen des Ciliarkörpers mit außerordentlich zahlreichen, in typischer Weise angeordneten Mitochondrien angefüllt sind, also zweifellos ein a usgesprochenes voll entwickeltes Chondriom besitzen, das an Deutlichkeit hinter dem vergleichsweise untersuchten der Epithelzellen der Aderhautgeflechte ${ }^{4}$ ), die ja entwicklungsgeschichtlich den Ciliarfortsätzen gleichstehen ${ }^{5}$ ) und die bekanntlich nach der heute allgemein

1) Methode nach Regaud. Fixation kleiner lebenswarm entnommener Gewebsstückchen in einer Mischung Formol (20 Vol.) und $3^{1} / 2$ proz. Bichromatlösung (80 Vol.) 4 Tage lang (Wechsel der Flüssigkeit alle 12 Stunden). Beizung in Kaliumbichromatlösung 31\% (8-14 Tage) lang. Danach Auswaschen in fließendem Was. ser (24. Stunden lang), aufsteigenden Alkohol, Xylol, Paraffin, sehr dünne Schnitte $(3 \mu)$. Färbung nach Heidenhains Hämatoxylin - Eisenlack - Methode. Über zweckmäßiges Vorgehen dabei siehe Meves, Arch. f. mikr. Anat. Y0. 1907.

$\left.{ }^{2}\right)$ Methode nach Schridde. Müller Formol 2 Tage lang (wechseln!); Müller: 2 Tage; fließendes Wasser, 24. Stunden. Osmiumsäure 1\%, 24 Stunden; Auswaschen mit destilliertem Wasser 24 Stunden; aufsteigender Alkohol. Paraffin.

3) Methode nach O. Schulze, vgl. Stöhr-Schulze, Lehrb. d. Histologie. 16. Aufl. 1915. S. 32, 33 ,Osmiumhämatoxylinmethode" (Stückfärbung).

4) Schon um 1700 wurde der Plexus chorioideus von Nuok als Glandula bezeichnet, später trat L u sch ka (,die Aderhautgeflechte des Gehirns", Berlin 1855) besonders für diese Auffassung ein. P. Ernst fand zuerst jm Aderhautepithel eine granuläre Struktur (Deutsche pathologische Ges. VII., 1904 und Zieglers Beiträge z. allg. Path. u. pathol. Anat. Suppl. VII; 1905) und wies ihr zunächst wegen der Ahnlichkeit mit der Struktur der Nierenzelle sekretorisohe Funktion zu, was durch spätere experimentelle Untersuchungen von ihm und Schlae pfer bestätigt wurde. - Der in einer Diskussionsbemerkung (gelegentlich der von mir im mediz.-naturwissensehaftlichen Verein in Heidelberg gehaltenen Vorträge über den Flüssigkeitswechsel im Auge) von Herm Geh. Rat Ernst erfolgte Hinweis auf diese Befunde veranlaßten mich zu den vorliegenden Untersuchungen. Vgl. auch Grynfelt und Enzi ère. Recherches cytologiques sur les cellules épithéliales des plexus chorioides. Extrait des comptes rendus de l'Association des Anatomistes 1912. Rennes.

5) Die Aderhautgeflechte der Hirnhöhlen bestehen aus einer spärlichen bindegewebigen Stroma mit reichlichen Blutgefäßen, mit einem einfachen Úberzug von epithelialen kubischen Zellen. Letztere sind die unveränderte Wandung der ursprünglichen epithelialen primären Vorder- bzw. Hinterhirnbläschen, da es an diesen Stellen weder zur Entwicklung von nervöser Substanz noch von Glia kommt, wie das an anderen Stellen der Fall ist (Corpus striatum, Ammonshorn usw.). Entwicklungsgesehichtlich entstehen die Aderhautgeflechte dadurch, daß die bindegewebige und blutgefäßreiche weiche Hirnhant in den Hohlraum der primären 
geteilten Ansicht die Absonderung der, dem Kammerwasser in der chemischen Zusammensetzung fast.. völlig gleichenden Cerebrospinal: flüssigkeit besorgen, in keiner Weise zurückbleibt. Dagegen konnte ich an der Irisvorderfläche nach keiner Methode auch nur eine Andeutung mitochondraler Bildungen wahrnehmen, und zwar an denselben Schnitten, was besonders beweisend ist, an denen die Mitochondrien im Ciliarepithel aufs deutlichste hervortraten.

Ebenso erwiesen sich die Epithelzellen der Hornhaut, die ich nur vergleichsweise, als Beispiel eines typischen Deckepithels, in den Kreis meiner Untersuchungen zog, nach den verschiedenen 'Methoden, "frei von Mitochondrien, wie dies zu erwarten war.

Wird allein sehon durch den Nachweis vom Vorhandensein eines voll entwickelten Chondrioms im Ciliarepithel seine sekretorisehe Funk. tion bewiesen, ebenso wie sein Fehlen an der Irisworderfläche eine solche Funktion hier auszuschließen erlaubt, so spricht die weitere sehr wichtige Tatsache durchaus in demselben Sinne, daß sich im Ciliarepithel, und zwar nur hier, eine Reihe von bemerkenswerten Eigentümlichkeiten im histologischen Bilde finden, die den an anderen Drüsenzellen und besonders am Plexus chorioideus beobachteten, wie ich, feststellte, prinzipiell fast völlig gleichen.

Ich fand, nicht in allen, aber in zahlreichen Zellen des Ciliarepithels, kleine Bläschen oder Tröpfchen von verschiedener Größe und Anzahl, die meist nach der freien Zelloberfläche zu, jedoch auch neben und seltener unterhalb des Kernes lagen. Eis finden sich Zellen mit zahl. reichen kleineren Bläschen, desgleichen solche mit einigen größeren Hohlräumen oder Vakuolen, die dann aufgebläht erscheinen und an denen man dann eine Armtt an Mitochondrien wahrnimmt.

Weiter beobachtete ich an solchen Präparaten, bei denen es gelungen war, die Zonulafasern im Zusammenhang mit den Ciliarepithelzellen zu erhalten, daß nahe der freien Zelloberfläche zwischen den Zonulafasem, also auBerhalb der Zellen, in der Hinterkammer ziemlich zahlreiche

Vorder- and Hinterhimbläschen himeinwwohert (im 2, bis 3. Monat beim Menschen), indem sie die dinne Epitheldecke vor sich her treibt und einfaltet (vgl. O. Hertwig, Elemente der. Entwicklungslehre des Menschen und der Wirbeltiere. 1916). Die Ciliarfortsätze bestehen aus einem spärlichen bindegewebigen Stroma mit reichlichen BlutgefäBen, mit doppeltem Utberzug von epithelialen kubischen und zylindrischen Zellen. Letztere sind die unveränderte Wandung der ursprüng. lichen epithelialen (sich aus der seitlichen Wand der primären Vorderhirnblasen ausstülpenden) Augenblasen, da es an dieser Stelle weder zur Entwichlung ron nervöser Substanz nooh von Qlia kommt, wie das an anderen Stellen der Fall ist (Netzhaut). Entwicklungsgeschichtlich entstehen die Ciliarfortsätze dadurch, daß die bindegewebige GefäBhaut in den Hohlrawm der: bereits zum Augenbecher eingestülpten. Augenblase hineinwuchert (im 2. bis 3. Monat beim Menschen), indem sie die infolge eines intensiven FI"̈chenwahstums entstehenden Falten beidex" Epithelblätter ausfulliț 
Tröpfchen wahrnehmbar waren, von meist homogener Beschaffenheit, die sich einige Male als aus einer Anzahl kleinerer Tröpfchen zusammengesetzt erwiesen. Meist hatten diese Tröpfchen im Osmiumpräparat mattgelbliches Aussehen, genau wie der Inhalt der intracellularen Bläschen und Tröpfchen, doch konnte an wenigen dieser Tröpfchen eine feinste blaue Körnelung ganz vom Aussehen der intracellularen Mitochondrien wahrgenommen werden.

Fin Blick auf die Textabbildungen 1 und 2 (S. 198) sowie auf Abb. 2-9 auf Tafel III wird das eben Gesagte veransehaulichen.

Textabb. 1 zeigt ein Mikrophotogramm (C. Zei B, Hommogen. Imers. Apochromat. 2,0 mm, Apert. 1,30 Comps. Ocular 4) eines Ciliarfor tsatzes der Katze nach der Methode von Rega ud. Man erkennt sehr deutlich die massenhaften feinen Körnohen, die in sämtlichen Zellen sehr reichlich vorhanden sind.

Textabb. 2 zeigt die Hornhaut desselben Tieres nach derselben Methode behandelt in gleicher Vergrößerung (500fach). Man findet die Zellen frei von Körnern, wie das bei einem Deckepithel ohne sekretorische Funktion zu erwarten war. (Vgl. Bericht der ophthalmolog. Ges. XLI. Taf. III, Abb. 1 u. 2.)

Abb. 2 (Zeichnung, Tafel III) zeigt 3 Zellen des Ciliarepithels von albinotischen Kaninchen nach der Methode von O. Sehulze. Man erkennt die zahlreichen in charakteristischer Weise zu Fäden angeordneten Körner im Protoplasma und sieht außerdem sehr deutlich einige Bläschen oder Tröpfchen (C. Zeiß, Homogen. Immers. Apochromat. 1,5 mm Apert. 1,30 Oeular 6. 1000 fache Vergrößerung) ${ }^{1}$ )

Abb. 3 (Zeichnung, Tafel III) zeigt 2 Zellen des Ciliarepithels der Katze nach der Methode von Regaud. Man sieht besonders in der rechten Zelle wieder zahlreiche Mitochondrien und kann ferner wahrnehmen, daß dieselben weniger zahlreich sind in der linken Zelle, die dafür aber in größerer Anzahl kleine Tröpfchen oder Bläschen enthält.

Abb. 4 (Zeichnung, Tafel III) zeigen eine Anzahl Zellen des Ciliarepithels bei albinotischen Kaninchen nach der Methode von Rega ud.

Man erkennt hier sehr deutlich

1. den außerordentlich starken Untersehied in der Färbbarkeit der Kerne, seit Heidenhain ein anerkanntes Charakteristikum der Drüsenzellen²),

2. die verschiedene. Gestalt und Größe der einzelnen Zellen und ihren verschiedenen Gehalt an Mitochondrien.

3. diè kleinen Tröpfchen außerhalb der Zellen im Maschenwerk der Zonulafasern, die genau denselben Farbenton zeigen wie der Inhalt der intrazellulären Bläschen, die an einer Zelle deutlich nach der freien Oberfläche zu sichtbar sind.

Abb. 5 (Zeichnung, Tafel III) zeigt 2 Zellen des Ciliarepithels der Gans nach der Methode von Regaud. Man sieht die zahlireichen zu deutlichen Fäden angeordneten Körner im Protoplasma.

Abb. 6 (Zeichnung, Tafel III) zeigt 2 Zellen des Ciliarepithels der Gans nach der Methode von Rega ud. In der linken Zelle findet man eine fädige Protoplasmastruktur, in der rechten dagegen nur wenige Körner, dafür aber nach der freien Oberfläche zu kleine Vakuolen.

1) Die folgenden Bilder sind bei derselben Vergrößerung gezeichnet. Für die Untersuchung benutzte ich das angegebene Objektiv meist mit Comps. Ooular 4 (etwa 700 fache Vergrößerung).

2) Über den Anteil des Kerns am SekretionsprozeB vgl. Dues berg, 1. c. S. 790; desgl. Heidenhain und Mawas, l. c. 


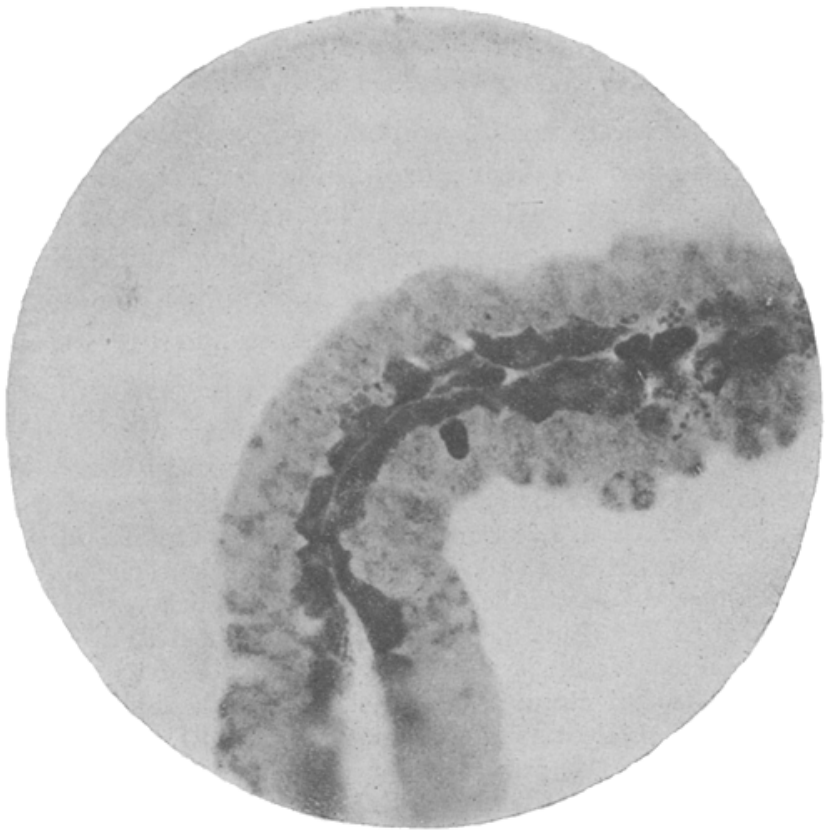

Abb. 1.

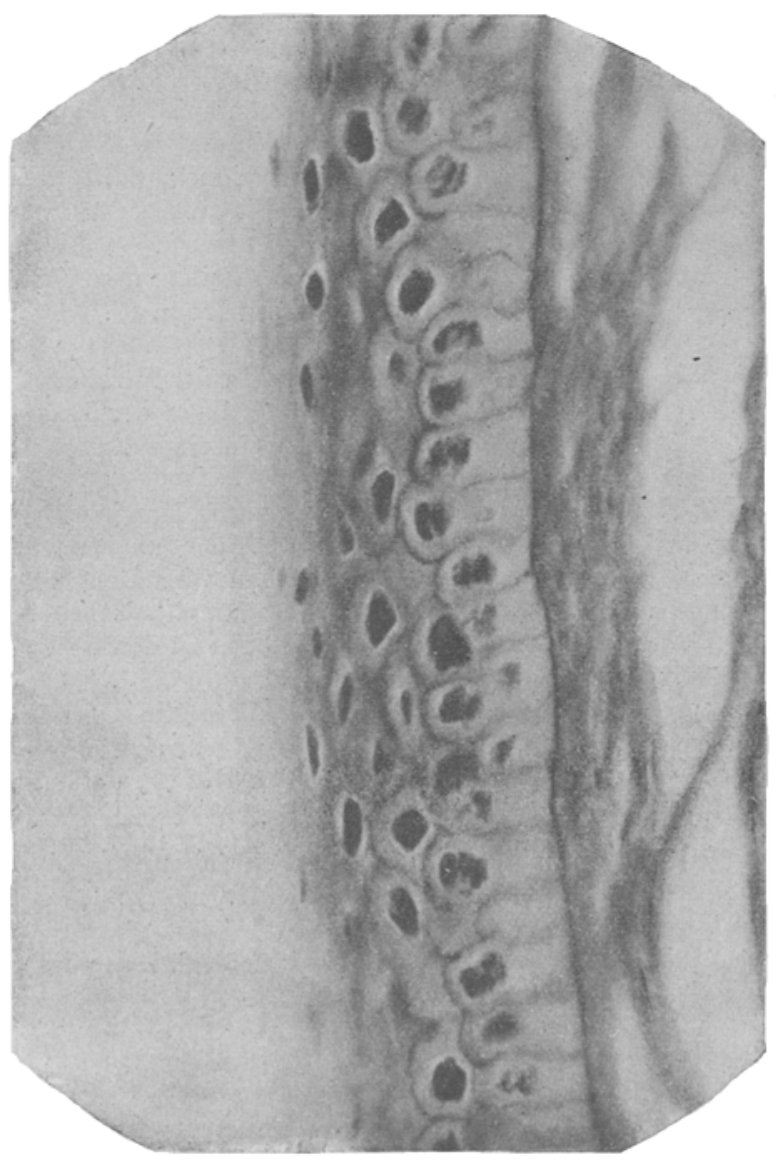

Abb. 2. 
Abb. 7 (Zeichnung, Tafel III) zeigt 1 Zellen des Ciliarepithels der Gans naeh der Methode von Regaud. Von den beiden äußeren Zellen zeigt die linke wieder fädige Protoplasmastruktur, die rechte einzelne Körner im Protoplasma.

In den beiden mittleren Zellen findet man nur sehr wenige Körner, dafür aber nahe der freien Zelloberfläche kleine Vakuolen. Die mehr nach der Zellbasis zu liegenden Vakuolen zeigen eine mit Eisenhämatoxylin dunkelgefärbte Hälle, die unmittelbar an der freien Zelloberfläche liegenden und die Zellmembran stellenweise leicht vorbuokelnden lassen diese gefärbte Hülle vermissen.

Wie die verschiedenen Bilder ${ }^{1}$ ) zeigen, findet man dicht nebeneinander vakuolenfreie und vakuolenhaltige Zellen. Hieraus geht hervor, daß diese Vakuolen nicht etwa Kunstprodukte darstellen, da man sie sonst in allen Zellen gleichmäBig tinden müBte, was nicht der Fall ist ${ }^{2}$ ).

Wenn man sich eingehender mit dem Studium dieser sehr interessanten Verhältnisse befaßt, so ist man verwundert über die großen morphologischen Variationen zwischen den allgemein für gleichartig gehaltenen Zellen des Ciliarepithels, die sich mit den verschiedensten von mir angewandten mitochondrialen Methoden in gleicher Weise zur Anschauung bringen lassen, und man kann sich der Auffassung nicht entziehen, in den beobachteten, eben in den Haupttypen geschilderten Struktureigentümlichkeiten der verschiedenen Zellen, den Ausdruck verschiedener Stadien funktioneller Zelltätigkeit zu erblicken.

Vergegenwärtigt man sich die bisherigen gesicherten Frgebnisse der Mitochondrienforschung für die cellaläre Drüsenphysiologie, so wird man die eben kurz geschilderten mikroskopischen Bilder so deuten müssen, daß diejenigen Zellen, die eine ausgesprochene, annähernd in der Längsrichtung der Zelle verlaufende fädige, rosenkranzartige Anordnung ihrer Körner zeigen, Zellen bei Beginn ihrer sekretorischen Arbeit darstellen. Aus den Bildern, in denen man eine Verringerung der nicht mehr fädig angeordneten Mitochondrien erkennt bei gleichzeitig vorhandenen mehr oder wenig zahlreichen, meist nach der freien Zelloberfläche liegenden kleinen Vakuolen, die teilweise noch von einer (mit mitochondralen Methoden) färbbaren Hülle umgeben sind, wird man auf die Umwandlung dieser Körner in kleine Tröpfchen schließen dürfen und die Bilder, auf denen man wahrnimmt, wie die kleinen Tröpfchen nahe der freien Zelloberfläche angeordnet liegen und die Zellmembran stellenweise leicht vordrängen, sowie aus den einzeln stark vorgebuckelten, kolbig aufgetriebenen (nicht färbbaren, d.h. nicht mitochondrienhaltigen) Zellenden, kann man wohl durch das Bestreben des verflüssigten Zellinhaltes erklären, die Zelle durch die freie Zelloberfläche hindurch zu

1) Abb. 2, 3 and 4 wurden von Fran E. Gutermann, Abb. 1, 5, 6, 7, 8 und 9 von Herrn Dr. Schl a efke, Assistenzarzt d. Heidelberger Augenklinik, gezeichnet.

2) Ich möchte bei dieser Gelegenheit kurz erwähnen, daß von mir auch das Pecten des Vogelauges mit mitochondralen Methoden untersucht wurde. Ich konnte kein Chondriom der entsprechenden Zellen nachweisen, was gegen eine sekretorische Funktion dieses Organs sprechen würde. 
verlassen. Wir hätten somit in den uns mikroskopisch als Vakuolen entgegentretenden Gebilden kleine Tröpfchen zu erbljcken, die anscheinend durch Verflüssigung und Zusammenfließen der Mitochondrien sich bilden und die aus physiologischem Kammerwasser bestehen würden, das wohl physiologischerweise durch Filtration durch die Zellmembran die Zelle verläßt, was man aus der meist intakt befundenen Zellmembran entnehmen darf. Der Inhalt der Vakuolen stellt offenbar das Aquivalent der in echten Drüsenzellen beobachteten Sekretkörner dar. Die Tatsache, da $B$ der Vakuoleninhalt der Ciliarepithelien (ebenso wie der im Epithel des Plexus chorioideus) sich nicht färbt (oder wenigstens nach Osmiumbehandlung nur einen ganz matten grauen Farbenton annimmt), im Gegensatz zu den sich färbenden Sekretkörnern echter Drüsenzellen, erklärt sich sehr einfach aus dem fundamentalen chemischen Unterschiede des Humor aqueus gegenüber echter Drüsensekrete, worauf ja Leber, wie schon besprochen, so nachdrücklich hingewiesen hat.

Um die physiologisch interessante Frage zu beantworten, ob das Kammerwasser ausschließlich als verflüssigter Tropfeninhalt ausgeschieden wird, oder ob, auch Mitochondrien selbst aus der Ciliarepithelzelle austreten, wie bei granulären Sekretionsvorgängen, ein Gedanke, der mir durch einige mikroskopische Bilder nahegelegt wurde, habe ich bei lebenden albinotischen Kaninchen Untersuchung von Kammerwasser mit starken mikroskopischen Vergrößerungen unter Zuhilfenahme der Dunkelfeldbeleuchtung vorgenommen.

Ich konnte im normalen Kammerwasser vereinzelte, sich teilweise in Bewegung befindliche (Brownsche Molekularbewegung), stark licht- brechende Körnchen von der Größe der Mitochondrien nachweisen, die nach künstlichen Ciliarkörperreizen (Muscarin, Pilocarpin, Eserin) eine deutliche Zunahme erkennen ließen.

Ich bin auf Grund dieser Befunde zu der Anschauung gelangt, daß das Kammerwasser unter physiologischen Verhältnissen in der Hauptsache als Flüssigkeit das Ciliaropithel verläßt und daß, falls Mitochondrien in nennenswerter Menge mitausgeschieden werden, sich die meisten derselben sehr bald verflüssigen müissen. Wird der Selrretionsprozeß lebhafter, dann können in größerem Ausmaße sich erst nach dem Austritt aus der Zelle verflüssigende Mitochondrien ausgeschieden werden ${ }^{1}$ ).

I) Nach lotaler ( 1 proz.) Eserinanwendung fand ich bei albinotischen Kaninchen bei der mikroskopischen Untersuchung des Kammerwassers (C. Zeiß A pochromat 4,0 Apert. 0,95) außer vermehrten Könchen gelegentlich vereinzelte ovaläre Zellkerne der Ciliarepithelien, an denen zahlreiche ebensolche Körnchon (Mitoohondrien) haftieten. -

Die Beantwortung der Frage, ob den im Kammerwasser nachgewiesenen Mitochondrien eine Bedeutung für die Pathologie des Auges zukommt, muß späteren Untersuchungen vorbehalten bleiben. 
Weiterhin habe ich nach vorausgeschickten intraperitonealen Pilocarpininjektionen bei Katzen und Kaninchen mikroskopische Untersuchungen des Ciliarepithels in frischem Zustande sowie nach Behandlung mit mitochondralen Methoden (besonders der von Rega ud) vorgenommen.

Da dureh Pilocarpin bekanntlich alle Drüsen in eine gesteigerte sekretorische Tätigkeit versetzt werden, so stand zu erwarten, daß in den Ciliarepithelien, wenn diese (wie ich das schon aus dem normalen Strukturbild ihres Protoplasmas mit Sicherheit entnommen hatte) wirklich eine physiologische sekretorische Funktion besitzen, diese durch Pilocarpin ebenfalls eine Steigerung erfahren würde, was im mikroskopischen Bilde in ähnlicher Weise zum Ausdruck kommen mußte, wie dies bei echten Drüsenzellen bereits bekannt ist.

Der positive Ausfall dieser Untersuchungen mußte also imstande sein, die Richtigkeit der von mir gegebenen Deutungen der im normalen Ciliarepithel festgestellten Zellstrukturen und somit die physiologische sekretorische Funktion des Ciliarepithels direkt zu beweisen.

Die Ergebnisse dieser Untersuchungen entsprachen durchaus den Frwartungen.

Ich fand bei der mikroskopischen Untersuchung der Ciliarepithelien von vorher mit Pilocarpin ${ }^{1}$ ) behandelten Tieren alle bei einer künstlich vermehrten sekretorischen Aktivität in echten Drüsenzellen bekannten und beschriebenen Veränderungen an Protoplasma und Kern in den Ciliarepithelien mit großer Deutlichkeit vor (vgl. Abb. 8 u. 9 auf Tafel III) ${ }^{2}$ ).

Am auffallendsten war die ganz beträchtliche Vermehrung der intracellulären Vakuolen.

Die Vakuolen waren fast in allen Zellen vorhanden, und zwar in gegenüber der Norm erheblich vermehrter Anzahl und Größe. Sie lagen meist nach der freien Zelloberfläche zu und verliehen vielen Zellen ein ausgesprochen aufgeblähtes Aussehen.

1) Katzen von $2700 \mathrm{~g}$ Körpergewicht erhielten zweimal je 1 ecm Pilocarpin 2 proz. mit 4 stündigem Zwischenraum intraperitoneal injiziert. Tötung durch Verbluten aus den Halsgefäßen 5-6 Stunden nach der ersten Injektion. Zur mikroskopischen Untersuchung im lebenswarmen Zustande benutzte ich albinotische Kaninchen, die bei einem Gewicht von $500 \mathrm{~g}$ Körpergewicht zweimal mit 4 stündigem $Z$ wischenraum je $0,5 \mathrm{ccm}$ einer 2 proz. Pilocarpinlösung intraperitoneal erhielten. Tötung der Tiere durch Dekapitieren 5-6 Stunden nach der ersten Injektion. Refraktometrisch findet sich nach dieser Zeit im Kammerwasser eine leichte Erhöhung des Brechungsindex (1,3357-1,3359) und eine geringe Erhöhung des EiweiBgehaltes $(0,06-0,09 \%)$. Man erkennt mit starken VergröBerungen an kleinen mit tangentialen Scherenschlägen abgetragenen Stückchen der Ciliarepithelien (die man am besten im Kammerwasser untersucht) sehr dentlich zahlreiche kleine intracellulär gelegene Tröpfchen.

2) Abb. 8 und 9 stellen Ciliarepithelien der Katze dar (Methode Regaud) nach vorherigen Pilocarpininjektionen. Abb. 9 zeigt zwei Zellen der Pars plana des Ciliarkörpers. 
Dabei beobachtete ich wieder genau wie im Ciliarepithel vorher intakter Tiere, daß die näher der Zellbasis gelegenen Bläschen in der Regel eine gefärbte, manchmal deutlich aus kleinen Körnchen bestehenden Hülle besaßen, die jedoch an denen mehr nach der freien Zellober. fläcche zu liegenden nur undeutlich oder überhaupt nicht zu erkenren war.

Weiterhin stellte ich fest, daR gerade die stark vakuolenhaltigen, mit zahlreichen Tröpfchen angefüllten, sich manchmal in die Hinterkammer weit vorwölbenden Zellen nur sehr wenige, anscheinend regellos angeordnete, verhältnismäßig schwach gefärbte Mitochondrien enthielten, und da. im Gegensatz dazu in anderen Zellen, ohne oder mit nur wenigen Vakuolen, sich zahlreiche, dicke, intensiv gefärbte, parallel der Längsrichtung der Zelle angeordnete Stäbchen fanden, an denen eine Zusammensetzung aus einzelnen Körnern nicht zu erkennen war.

Aber auch völlig mitochondrien- und vakuolenfreie Zellen waren vorhanden, die meist eine geringere Zellhöhe besaßen.

Sehr auffallend waren auch die von mir (bei den gewählten Pilocarpindosen und Zeitverhältnissen) festgestellten Veränderungen an den Zellkernen.

Der am intakten Auge so überaus deutliche Unterschied in der Färbbarkeit der Kerne war nach Pilocarpin so gut wie völlig verschwunden.

Fast alle Kerne waren einander vollkommen gleich und erschienen außerordentlich bla $B^{1}$ ). Im Innern dieser blassen, etwas aufgeblähten Kerne sah man mit größerer Deutlichkeit meist zweì etwas vergrößerte intensiv gefärbte Kernkörperchen. Weiterhin bemerkte man bei einer Anzahl von Zellen im Innern des Kernes außer den Kernkörperchen noch einige feine dunkelgefärbte Körnchen, die meist der Kernmembran innen dicht anlagen.

Die blassen Kerne, vor allem aber die gegen die Norm innerhalb der Zellen viel zahlreicheren kleinen Tröpfchen gaben dem mikroskopischen Bilde des Ciliarepithels nach. vorausgegangener Pilocarpinvergiftung des betreffenden Tieres ein so charakteristisches Aussehen, daß man unter dem Mikroskop das Ciliarepithel eines intakten und eines mit Pilocarpin vorbehandelten Tieres mit Leichtigkeit unterseheiden konnte.

Die eben beschriebenen Veränderungen nach Pilocarpininjektion fanden sich auch in den Epithelzellen der Pars plana des Ciliarkörpers sehr deutlich ausgesprochen (Abb. 9 auf Tafel IIr).

An der Vorderfläche der Iris konnten jedoch nach Pilocarpin nicht die geringsten Veränderungen gegenüber der Norm festgestellt werden.

Aus den geschilderten Befunden geht hervor, daß man nach Einverleibung von Pilocarpin, das bekanntlich einen spezifischen sekretorischen Reiz auf alle Drüsenzellen ausübt, im Ciliarepithel (bei Anwen-

- $)$,Daroh Sekretion ersehöpfte Zellen sind am chromatinarmen Kern zu erkennen." P. Ernst (1. c.). 
dung derselben Reagenzien) ausgesprochene Veränderungen gegenüber der Norm im Verhalten des Kernes und der Protoplasmastruktur feststellen kann.

Diese vor und nach Pilocarpin an denselben Zellen mit denselben Reagenzien erhaltenen verschiedenen Bilder müssen notwendigerweise verschiedenen Zuständen dieser Zellen im Leben entsprechen.

Die festgestellten Veränderungen gleichen ganz und gar den durch dieses Gift in echten Drüsenzellen beobachteten und beschriebenen, die allgemein auf eine vermehrte sekretorische Tätigkeit bezogen werden.

Durch diese eben mitgeteilten mikroskopischen Befunde im Ciliarepithel nach Anwendung eines alle Drüsen zu vermehrter Tätigkeit anregenden Mittels (Pilocarpin) wird die physiologische sekretorische Funktion dieser Zellen klar' bewiesen.

Da an der Trisvorderfläche im Gegensatz hierzu nicht die geringsten Veränderungen nach Pilocarpinreiz auftreten, ist eine sekretorische Funktion der Irisvorderfläche hiermit ausgeschlossen.

Aus den geschilderten, mit mitochondralen Methoden im Ciliarepithel völlig intakter Tiere festgestellten Protoplasmastrukturen, die durch vorherige Behandlung der Tiere mit Pilocarpin in charakteristischer Weise verändert werden, und die, wie gezeigt, große Ähnlichkeit mit den in echten Drüsenzellen und im Epithel der Aderhautgeflechte $^{1}$ ) vorhandenen aufweisen, da wir auch im Ciliarepithel in

1) Der Parallelismus zwischen Corpus ciliare und Plexus chorioideus tritt nach folgenden Richtungen hin zutage: 1. Entwicklungsgeschichtlich (vgl. S. 195, Anm. 5); 2. anatomisch (Protoplasmastruktur der Epithelien, Sekretvakuolen). Beschaffenheit der Kerne (große, regelmäßige, ovuläre, nach der Zellbasis zu gelegene Kerne mit stark wechselndem Chromatingehalt und großen Kernkörperchen); 3. funktionell a) Färbung durch vitale Farbstoffe (nach Isaminblau Farbstoffkörnchen innerhalb der Ciliarepithelien); b) relatives Zurückhalten vitaler Farbstoffe durch die Epithelien, die nur in Spuren in das betreffende $A b$ sonderungsprodukt übergehen (Goldmann, Kafka und eigene Beobachtungen). c) Gleichheit ihrer physiologischen Sekretionsprodukte in chemischer und physikalischer Beziehung. (Minimaler EiweiBgehalt, leichte Erhöhung des Kochsalzgehaltes gegenüber dem Blute, wechselnder osmotischer Druck (vgl. v. d. Hoeve, v. Graefes Arch. 82. 1912; Reichmann, Dtsch. Zeitschr. f. Nervenheilk. 42. 1911).

Aber auch unter pathologisehen Verhältnissen findet sich der Parallelislismus zwischen beiden Organen: 1. Nach druckherabsetzenden Punktionen Hyperämie der Gefäße (Meningismus nach Spinalpunktion, vgl. Reich mann, Zeitschr. f. d. ges. Neur. u. Psych. 11, Heft 25). 2. Der regenerierte Liquor cerebrospinalis zeigt ebenso wie der regenerierte Humor aqueus einen vermehrten EiweiBgehalt (eigene refraktorische und chemische Liquoruntersuchung nach Ventrikelpunktion bei chronischem Hydrocephalus durch Prof. Wilms (vgl. auch Reichman $n$, l.c.). 3. Bei entzündlichen Prozessen der GefäBhaut von Auge und Gehirn vermehrter Eiweißgehalt im Humor aqueus bzw. Liquor cerebrospinalis (Lues) Iritis. 4. Dem vermehrten Eiweißübertritt geht ein vermehrter Fluoresceinübertritt parallel (vgl. Kafka, Zeitschr. f. d. ges. Neur. u. Psych. 4 v. 9. 1910).

Diese Parallele läßt sich experimentell noch weiter führen, wie in späteren Mitteilungen gezeigt werden wird. 
gewissen Stadien ihrer sekretorischen Arbait deutlich einen basalen nutritiven und einen exkretorisehen Pol unterscheiden konnten, wobei wir in der Richtung von der Basis zur Oberfläche den Weg des Sekretionsvorgangs von der beginnenden Verarbeitung in den Mitochondrien bis zum fertigen Kammerwassertröpfchen ${ }^{1}$ ) erkannten, ergibt sich mit der größten Sicherheit, mit der überhaupt aus morphologischen Bildern auf physiologische Vorgänge geschlossen werden kann, die Beteiligung des Ciliarepithels beim Vorgang der Kammerwasserabsonderung. Wenn somit auch vom chemischen und physikalischen Standpunkte aus die Annahme vitaler Kräfte bei der Kammerwasserbildung nicht erforderlich erscheint (Th. Le ber), so sind doch die in den Ciliarepithelien beschriebenen anatomisohen auf funktionelle Tätigkeit zu beziehenden Bilder, die uns den Vorgang der Kammerwasserbildung enthüllen, allein nach physiko-chemischen Gesetzen ohne die Mitbeteiligung lebender Zellkräfte zur Zeit noch nicht verständlich.

Aus diesem Grunde haben wir im Vorgang der Kammerwasserabsonderung keinen einfachen mechanischen Prozeß zu erblicken, sondern einen komplizicrten, im kolloidalen System der Zelle mit Hilfe vitaler Kräfte sich abspieleuden Filtrationsvorgang unter gleichzeitiger Wirksamkeit von Diffusion und Osmose, so daß wir wohl berechtigt sind, das Ciliarepithel als ein aktives sezernierendes Epithel zu bezeichnen, das mit einem Drüsenepithel verglichen werden darf.

Weiterhin aber beweisen die erhobenen Befunde, daB auch im intakten Auge ein physiologischer SekretionsprozeB stattfindet, und" daß als Sekretionsorgan nicht die Irisvorderfläche, sondern der Ciliarkörper (einschließlich seiner Pars plana) betrachtet werden muB, da wir nur hier (und beim Kaninchen auf die Irishinterfläche übergreifend) die ihrer Protoplasmastruktur nach auf sekretorische Funktion hindeutende und daher mit Drüsenepithelien vergleichbaren Zellen lokalisiert finden.

Wenn somit Th. Leber ${ }^{2}$ ) sebon vor Jahren seine Meinung wiederholt dahin aussprach: „Die Absonderung des Kammerwassers ... muß einer Tätigkeit der Ciliarfortsätze zugeschrieben werden, zu welcher vielleicht auch die Hinterfläche der Iris einen kleinen Teil beiträgt", so müssen wir allein schon auf Grund der vorstehend geschilderten cellulären Befunde diese Ansicht nunmehr als bewiesen ansehen.

1) Daß diese an völlig intakten Augen intracell nlar gelegenen, aur an sehr dïnnen Schnitten mit starker VergröBerung stcbtbaren fleinen Bläschen nichts mit dem von Greeff nach Vorderkaramerpunktion gefundenen großen blasigen Abhebungen des Ciliarepithels zu tun haben, möchte ich hiermit ausdrücklioh betonen.

2) Die Zirkulations- und Ernährangsverhältnisse des Auges. Handb. GraefeSaemisch. 2. Aufl. 1903. S. 233. 


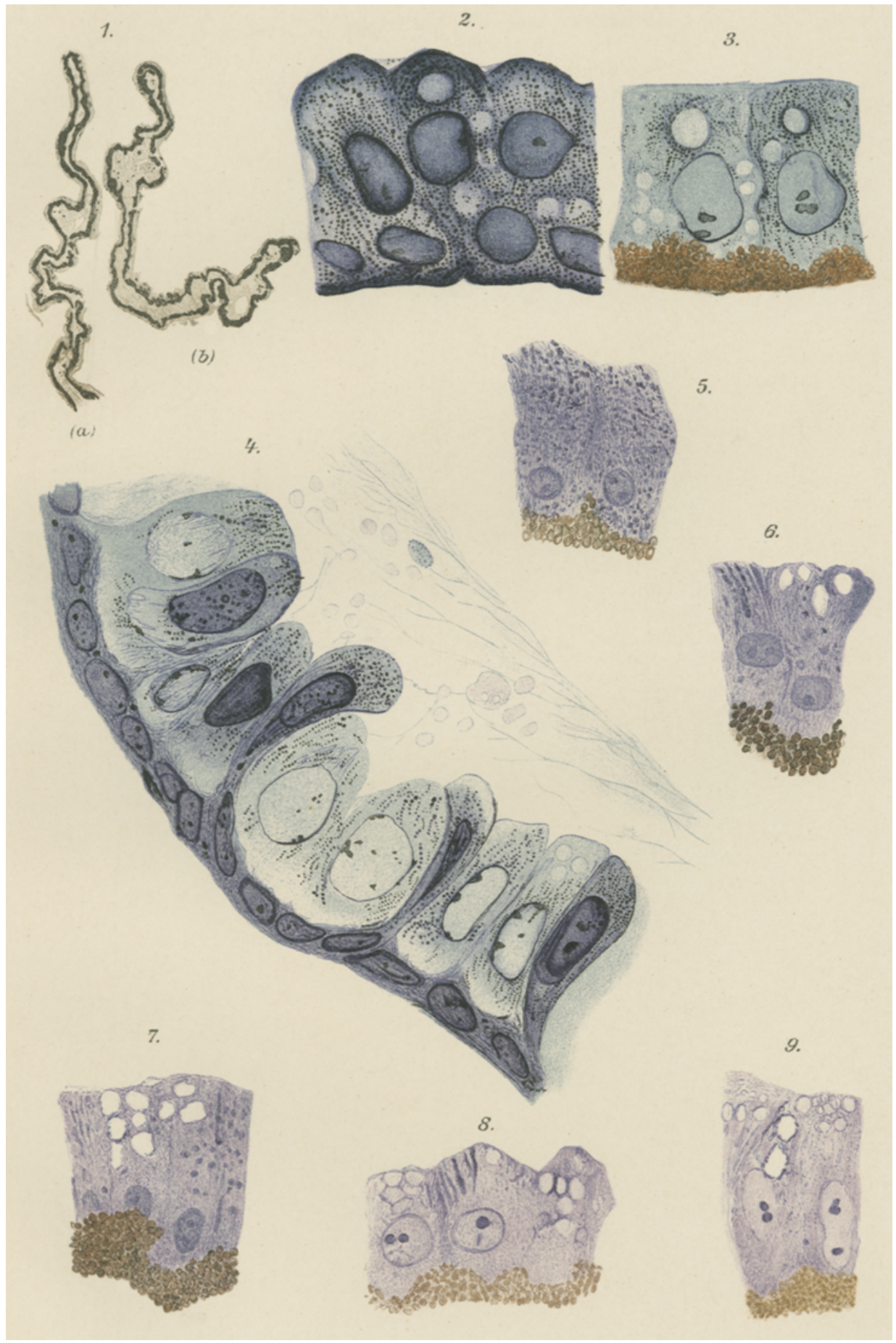

